\title{
The Use of Ultrasound Guided Pre-Operative Colloidal Charcoal Injection in the Surgical Management of Recurrent Differentiated Thyroid Cancer
}

\author{
Ali Moustafa ${ }^{1}$, Frederick Green ${ }^{1}$, Sheila Fraser ${ }^{1}$, Sriram Vaidyanathan ${ }^{2}$, and Jim Moor ${ }^{3,4}$ \\ ${ }^{1}$ Leeds Teaching Hospitals NHS Trust \\ ${ }^{2}$ Leeds Cancer Centre \\ ${ }^{3}$ University of Leeds \\ ${ }^{4}$ Leeds General Infirmary
}

July 16, 2020

\section{Title}

The Use of Ultrasound Guided Pre-Operative Colloidal Charcoal Injection in the Surgical Management of Recurrent Differentiated Thyroid Cancer

Keywords: thyroid cancer, neck recurrence, charcoal injection, ultrasound guidance, pre-operative localisation, reoperation

\section{Introduction}

Appropriately treated differentiated thyroid cancer (DTC) confers an excellent 10-year survival rate of over $90 \%$ for both papillary and follicular carcinoma ${ }^{1}$. However up to $30 \%$ of patients experience loco-regional recurrence within 10 years of diagnosis.

The routine use of post-treatment ultrasound (US) surveillance and thyroglobulin assay allows for early detection of non-palpable recurrent disease. Treatment options include radioiodine ablation or further surgical intervention; the former is limited to sub-centimetre lesions that exhibit radioiodine uptake. Recurrent disease may be iodine negative i.e. dedifferentiated in up to $30 \%$ of patients and may persist despite ablative treatment ${ }^{3}$. For these cases, surgical excision remains the mainstay of treatment.

The localisation of recurrent disease and identification of important structures to be preserved in previously dissected necks is challenging due to the presence of fibrotic scar tissue, neovascularisation and modified anatomy. The complication rates for transient and persistent hypoparathyroidism and recurrent laryngeal nerve injury are reportedly significantly higher in secondary vs primary surgery ${ }^{3}$.

Various techniques are employed for pre-operative localisation of non-palpable lesions in patients with cytologically proven recurrent DTC (rDTC) in the neck. These include intra-operative US guidance, $\mathrm{I}^{131}$ guided gamma probe and Methylene blue dye.

The use of charcoal tattooing for identification of recurrence of malignancy is well established in secondary breast surgery where it has been shown to be both safe and effective ${ }^{4}$ and in recent years, has been shown to be successful in the surgical management of $\mathrm{rDTC}^{5,6}$.

We aim to inform the readership of the technical aspects of using activated charcoal suspension injection for the localisation of DTC in the neck of previously treated patients based on our experience at [removed for blind peer review]. 


\section{Technical Description}

Appropriate patients are selected on the basis of having cytologically proven recurrent DTC that cannot be managed with ablative radioiodine and have small volume non-palpable recurrent disease in the neck at the site of previous surgery (either thyroid bed or neck dissection). The patient is subject to general anaesthesia, and prior to the skin incision the lesions are re-identified and confirmed using US. Between 0.5

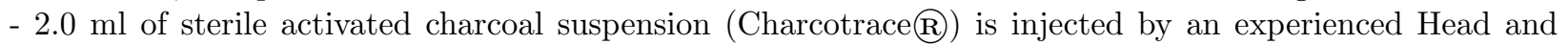
Neck radiologist under ultrasound guidance either into the lesion or the tissues immediately superficial to the target lesion. In addition, the lesion's relationship to anatomical landmarks and depth from the skin are imaged and reported to the operating surgeon.

Where possible the previous thyroidectomy/neck dissection incision is re-utilised and dissection is performed carefully towards the lesion which is now easily identified visually due to the obvious charcoal staining of the tissues immediately anterior to it, and the surgeon's knowledge of the site of the lesion relative to other anatomy. Once the lesion is excised, a repeat US can be performed intra-operatively to confirm excision before closure in equivocal cases. Recurrent laryngeal nerve monitoring is used if the lesion is in proximity to the nerve.

Fig.1 The recurrent lesion is easily identified due to the black hue of the charcoal dye anterior to its surface. Intra-operatively the lesion was impalpable but was confirmed by the presence of Charcotrace and pre/post resection ultrasound images, see figure 2 .

Fig.2 Pre-operative (left hand image) ultrasound scan (USS) demonstrates an irregular hypoechoic lesion medial to the carotid artery and lateral to the tracheal wall (yellow arrow) consistent with recurrent papillary thyroid cancer. The post-operative (left hand image) USS demonstrates the lesion has completely resected. CA: Carotid Artery, IJV: Internal Jugular Vein, TL: Tracheal Lumen

\section{Discussion}

The benefits of adopting this approach in the management of these patients are severalfold. Firstly, these small volume recurrences often offer no intra-operative tactile or palpable feedback to the surgical team, so the tissue staining provides clear visual feedback that the correct tissue has been encountered. Secondly, wide local resection of these lesions is then permissible with increased confidence that the primary objective of the surgery will be achieved, and thirdly this will be done with minimal risk to the RLN and parathyroid glands. Furthermore, the immediate post resection ultrasound image can provide additional radiological certainty of surgical success. This has been reported in the literature and a clear reduction of peri-operative complications has been achieved in salvage surgery for these patients ${ }^{5,6}$.

Charcoal suspension is readily available in [removed for blind peer review]. The radiological procedure does not require additional training for either the surgeon or radiologist. Additionally, the injection can be performed even a few days prior to the surgery due to long tissue retention. This may obviate the need for a radiologist to be available during surgery. Crucially, the charcoal tattooing does not interfere with histological analysis of the specimens obtained ${ }^{5,6}$, although it is good practice to alert the reporting histopathologist as to its use and location relative to the histopathology specimen.

Charcotrace $(\mathrm{R}$ is a $3 \mathrm{ml}$ sterile suspension containing $120 \mathrm{mg}$ of activated charcoal and sodium chloride in a $7 \mathrm{ml}$ vial. Activated Charcoal suspensions are known to be safe to use in this fashion with no allergic complications reported in the literature ${ }^{4,5,6}$.

In a case series of $12 \mathrm{rDTC}$ lesions excised using charcoal injection in South Korea, 1 patient had a temporary dark dot mark on the site of skin puncture when giving the injection ${ }^{5}$. Additionally, other injection associated complications reported in the literature include pain and haematoma formation. Thus, during the consent process for the procedure patients are informed that there is a small risk of a pin prick tattoo at the site of the needle entry, alongside the other risks of bleeding, infection, recurrent laryngeal nerve injury and hypoparathyroidism. 
The authors have provided a description of the technical process for the safe and effective use of ultrasound guided activated charcoal localisation for rDTC nodules in the neck. Our anecdotal experience is that this technique reduces operative time and post-operative complications in this cohort of patients although at time of writing we are not able to state this categorically.

\section{Key Points}

1. Injection of activated charcoal suspension facilitates visual confirmation of the site of lesion/s to be excised.

2. Use of activated charcoal suspension does not require additional training for radiologist or surgeon.

3. Injection of activated charcoal suspension into human tissue has a longstanding and well-established safety profile.

\section{References}

1. Dal Maso L, Tavilla A, Pacini F, Serraino D, van Dijk B, Chirlaque M et al. Survival of 86,690 patients with thyroid cancer: A population-based study in 29 European countries from EUROCARE-5. European Journal of Cancer. 2017;77:140-152. doi: 10.1016/j.ejca.2017.02.023

2. Mazzaferri E, Kloos R. Current Approaches to Primary Therapy for Papillary and Follicular Thyroid Cancer. The Journal of Clinical Endocrinology \& Metabolism. 2001;86(4):1447-1463. doi: 10.1210/jcem.86.4.7407

3. Durante C, Haddy N, Baudin E, Leboulleux S, Hartl D, Travagli J et al. Long-Term Outcome of 444 Patients with Distant Metastases from Papillary and Follicular Thyroid Carcinoma: Benefits and Limits of Radioiodine Therapy. The Journal of Clinical Endocrinology \& Metabolism. 2006;91(8):2892-2899. doi: $10.1210 /$ jc. $2005-2838$

4. Canavese G, Catturich A, Vecchio C, Tomei D, Estienne M, Moresco L et al. Pre-operative localization of non-palpable lesions in breast cancer by charcoal suspension. European Journal of Surgical Oncology (EJSO). 1995;21(1):47-49. doi: 10.1016/s0748-7983(05)80067-8

5. Kang T, Shin J, Han B, Ko E, Kang S, Hahn S et al. Preoperative Ultrasound-Guided Tattooing Localization of Recurrences After Thyroidectomy: Safety and Effectiveness. Annals of Surgical Oncology. 2009;16(6):1655-1659. doi: 10.1245/s10434-009-0431-7

6. Soprani F, De Vito A, Bondi F, Magliulo G, Rene S, Cappi C et al. Preoperative charcoal suspension tattoo for the detection of differentiated thyroid cancer recurrence. Molecular and Clinical Oncology. 2019;. doi: $10.3892 /$ mco.2019.1826 

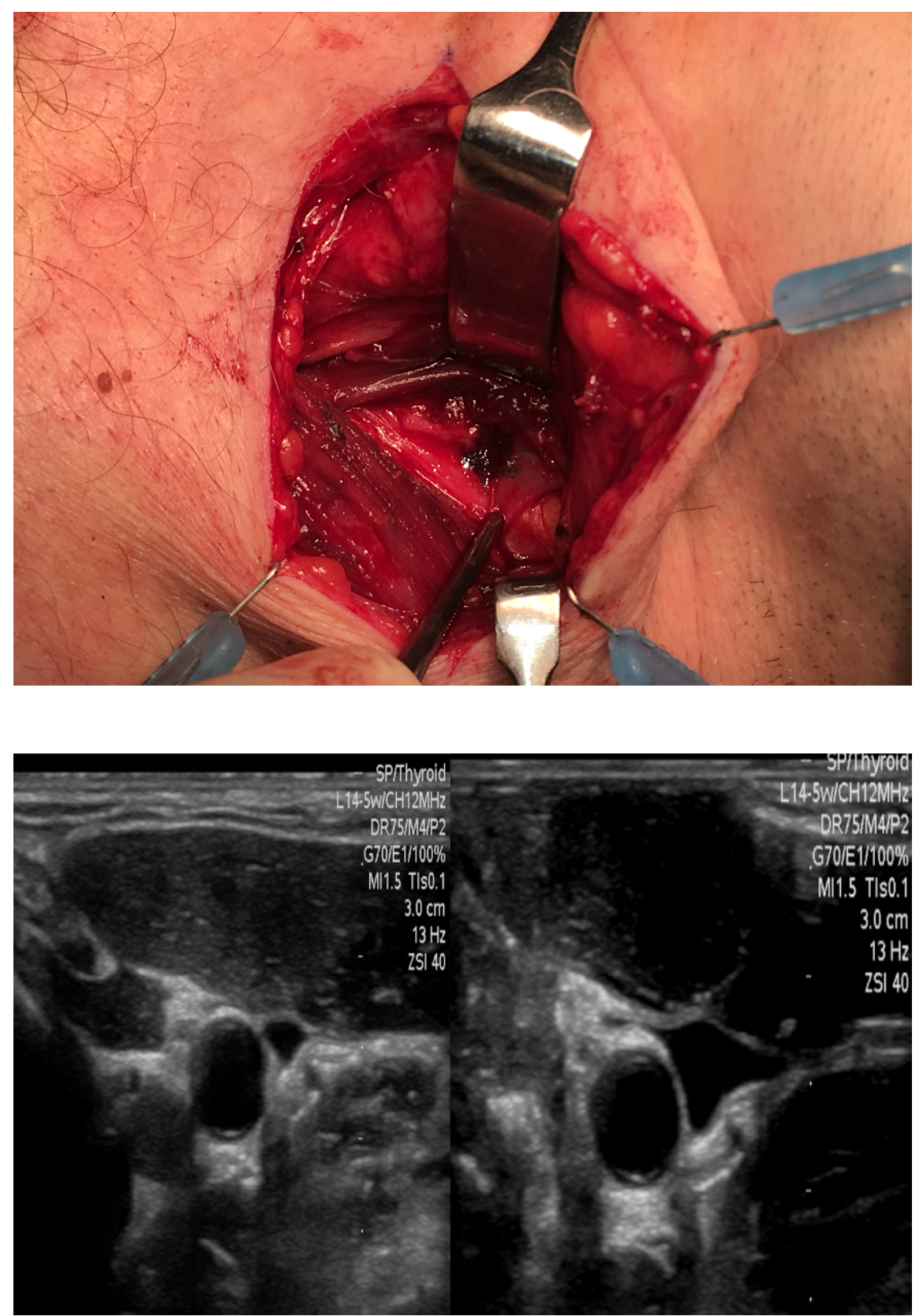